\title{
Recognising Emotions from Keyboard Stroke Pattern
}

\author{
Preeti Khanna \\ Faculty \\ SBM, SVKM's NMIMS \\ Vile Parle, Mumbai
}

\author{
M.Sasikumar \\ Associate Director \\ CDAC, Kharghar \\ Navi Mumbai
}

\begin{abstract}
In day to day life, emotions are becoming an important tool which helps to take not only the decisions but also to enhance learning, creative thinking and to effectively correspond in the social interaction. Several studies have been conducted comprising of classical human human interaction and human computer interaction. They concluded that for intelligent interaction, emotions play an important role. By embedding the emotions in the interaction of human with machine, machine would be in a position to sense the mood of the user and change its interaction accordingly. Hence the system will be friendlier to the user and its responses will be more similar to human behaviour. In general, human beings make use of emotions through speech, facial expression and gestures for conveying the crucial information. This paper presents an attempt to recognize selected emotion categories from keyboard stroke pattern. The emotional categories considered for our analysis are neutral, positive and negative. We have used various classifiers like Simple Logistics, SMO, Multilayer Perceptron, Random Tree, J48 and BF Tree, which is a part of WEKA tool, to analyse the selected features from keyboard stroke pattern.
\end{abstract}

\section{General Terms}

Emotion recognition, Classifier, WEKA

\section{Keywords}

Emotion categories, Human computer interaction, Classification Algorithms, Empirical study

\section{INTRODUCTION}

Recent advances in human-computer interaction indicate the need for user interfaces to recognise emotions of users while they interact with the computer. The possible applications of an interface capable of assessing human emotional states are numerous. One of the uses of such an interface is to enhance the sensitivity of an automatic tutor which can adjust the content of the tutorial and speed at which it is delivered. In the learning environment, some of the students express their emotions and are energetically engaged while some other students generally are not interested and timid. Knowing the student's emotions, computer agents can become more effective as a tutor. A computer agent can learn the student's preferences and offer better interactions. It is beneficial to those children who are timid and not interested in science or creative activities, with the aim to sustain a continuous interest. On the other hand, it is also helpful in encouraging those children who are already comfortable with the learning environment so as to explore further and accept more challenges. As in e-learning, other areas such as e-commerce, entertainment (video games, virtual reality, and training programs) etc. can also benefit tremendously from this direction of work. At the same time, many researchers acknowledge that affect has been overlooked by the computer community in general (Picard et al. 2002).

Many researchers have pointed out that there is a need of combining evidence from many modes of interaction so that a computer system can generate as valid hypotheses as possible about users' emotions (Oviatt 2003, Pantic et al. 2003). Many of the researchers had reported about audio and visual modalities which are used to recognise emotional state of the human. Visual modalities include facial expression, gesture and pose tracking.

Indeed, human emotions are not only expressed through faces, people's voices but also by people's actions (e.g., keyboard-stroke patterns). This view has been supported by many researchers in the field of human computer interaction (e.g. De Saliva et al. 1997, Busso et al. 2001, Pantic et al. 2003, Picard 2003, Oviatt 2003, Zimmermann et al. 2003, Virvou et al. 2007).

However very little work has been done in the literature towards the usage of keyboard while typing to know about the user's emotional state. In this paper we will be focusing on keyboard modality during the interaction of the end user with it to relate with his emotional state. First section of this paper discusses the related work done so far in this direction. Then we will be discussing the empirical study done and the details of our experiments to validate the results which we found as the outcomes of the empirical study.

\section{RELATED WORK}

In early 1960s the study had been done by psychologist Paul Ekman and colleagues (Ekman, 1982, 1992, 1999; Ekman et al.1994). This study resulted in the identification of a small number of so-called "basic" emotions, namely: anger, disgust, fear, happiness, sadness and surprise, with contempt added only recently. Ekman and Friesen $(1975,1977)$ developed the "facial action coding system (FACS)," a method for quantifying facial movement in terms of component muscle actions. For the past decade, there has been a significant amount of research done in the computer vision community on extracting facial actions, facial expressions, recognizing hand, head and body gestures - for example, in sign language recognition. According to $\mathrm{Wu}$ and Huang 2001, the detection and interpretation of hand gestures is becoming an important part of human computer interaction.

Zimmerman et al. 2003 proposed a system that monitors users' behaviour from standard input devices, such as the keyboard or the mouse. In their system, the analyzed parameters included the number of mouse clicks per minute, the average duration of mouse clicks (from the button-down to the button-up event), the maximum, minimum and average mouse speeds, the keystroke rate (strokes per second), the average duration of a keystroke 
(from the key-down to the key-up event) and performance measurements. George A. Tsihrintzis et al., 2008 included keyboard -stroke information in order to improve the accuracy of visual-facial emotion recognition. They found that the visualfacial modality may allow the recognition of certain states, such as neutral and surprise, with sufficient accuracy. However, its accuracy in recognizing anger and happiness can be improved significantly if assisted by keyboard-stroke information.

In this paper, we consider keyboard-stroke information as one of the modality to know about the emotional state of the end user. The keyboard is the most commonly used input device in a human-computer interaction session. Our work has been concentrated on three basic categories of emotions - positive emotional state, negative emotional state and the emotionless state which we refer to as neutral.

\section{EMPERICAL STUDY OF KEYBOARD STROKE PATTERN}

We explored interaction with keyboard as a source of information about the user's emotional state. We started our experiment with the empirical study to know whether keyboard stroke information could compliment the emotion recognition other than audio and visual modality. Our empirical study involved 300 users (45\% of Female and $55 \%$ of Male) of varying educational background, ages and levels of computer experience (see Figure 1 and 2).

Figure 1: Age distribution of the participants

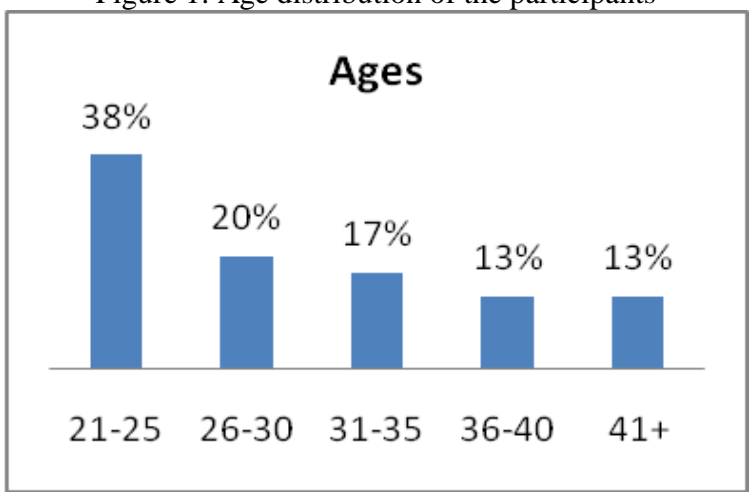

These users were given a small paragraph to read and then they are asked to note down the emotional state they feel after reading the paragraph along with the justification. Each of these users was asked to fill a questionnaire relating the typing pattern and their emotional state while typing. For example, under various emotional experiences like (neutral state, negative state comprising of disgust, contempt, angry, irritated, Restless, Bored, Sad, Dislike and positive state comprising of Affection Relaxed, Relief, Pleased, Delighted, Excited, Happy, Satisfied) what you felt while you were using the keyboard for typing. And they need to map with six basic actions such as (i) user types
Figure 2: Education level of the participants

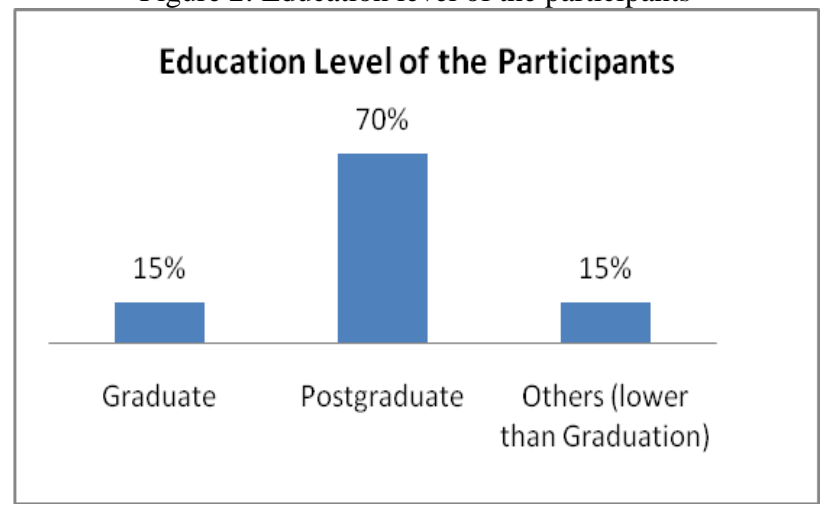

normally (ii) user types quickly (speed higher than the usual speed of the particular user) (iii) user types slowly (speed lower than the usual speed of the particular user) (iv) user uses the backspace key often (v) user hits unrelated keys on the keyboard (vi) user does not use the keyboard. The questionnaire includes the above mentioned six basic actions and the respondents need to be map their emotional states to these actions.

\subsection{Primarily Results from empirical study}

Most of the participants agree that when they are nervous the possibility of making mistakes is higher. This is also the case when they have negative feelings. Mistakes in typing followed by many backspaces and use of unrelated keys are usually seen. $88 \%$ of respondents agree that they will type normally when they are in neutral state. But when they are in negative emotional state like angry, disgust, irritated, bored, and sad their typing speed will reduce as compared to their neutral state. And $28 \%$ of respondents said that they will use more backspaces and $20 \%$ agree on relatively more usage of unrelated keys when they were in negative emotional state. $62 \%$ of respondents said their typing speed will increase relatively when they are in positive emotional state like happy, excited etc. It has been observed that usage of the backspaces and unrelated keys under positive emotional state reduces drastically (from $28 \%$ to $2 \%$ ).

\section{FRAMEWORK FOR EXPERIMENT}

Now to check these hypotheses in practice, we have some more experiments. We implemented a program to capture some of these parameters while a user is using the keyboard and we have tried to relate these parameters to the set of our defined emotional states. We have developed a simple software user interface (in JAVA) which more or less acts as an online recorder of user's keyboard behaviour. The basic function of this system is to capture all the data entered by the user using the keyboard of the computer. The data is recorded in a log file for our analysis along with relevant timing information. For our experiment, we requested male and female users from various organizations to participate according to their convenience. 
Total of 41 users (from our initial sample of 300) participated. We should have some document to type in our recorder so that we can capture the details of the parameters as defined above and correlate with their emotional states. For this, samples of paragraphs were chosen. We had chosen two set of paragraphs slightly different from each other for each of the emotion categories -neutral, positive and negative emotion as it could happen that if exactly the same text is given to these participants a number of times, they could memorize it and hence may distort the actual typing trend. These paragraphs consisting of 8 to 9 lines were used across all the participants to provide standardization.

We want to conduct this experiment under various emotional states of users as listed above and hence we need to run our experiment at different times depending upon participant's availability. It took about 4 to 5 months to collect the primary data across all the individuals. While running our experiment we assumed that participants know their emotional states though it may be possible that they manipulate their emotional states or misjudge them. We assumed that the input given by the participants about their emotional states were best to their knowledge.

Further studies have been done to know about the how emotions of users is going to depend on when he is interacting with the keyboard using WEKA tools. We extracted total of eight features such as typing speed, (mode, standard deviation, standard variance and range) of number of characters typed in 5 second interval, total time taken for typing, total number of backspaces used, and idle time in between (if any) when the user is not typing anything. All of them have given the test for typing in the prescribed text for three categories of emotional state: neutral, negative and positive. We need the WEKA software for carrying out the analysis. The basic objective was to see if any subset of these parameters would provide significant evidence towards a specific class of emotions. This required analysing the features collected for significance and promising the list if appropriate and choosing a suitable classifier which works best with the available data. This was crucial since collecting a large volume of reliable data is extremely difficult in this domain and hence classifiers which can learn well from a minimal data and which has a higher degree of tolerance for inconsistently in the data would be preferable.

\section{RESULTS}

Various classification algorithms - simple logistics, SMO, Multilayer Perceptron, Random Tree, J48, BF Tree were used to do the analysis with the help of WEKA. Some of the results are shown in the table 1 . These results indicate that negative emotional states are more visible and recognized better using the keyboard modalities. The change in the pattern of typing speed due to emotional state (either positive or negative) is seen and compared with reference to its corresponding neutral state.

Figure 3 and 4 (below) shows the variation of typing speed for negative and positive emotional state w.r.t. neutral state across our samples. It has been observed that some of the users while typing in experiments have been distracted by the outside world (in the form of phone calls etc). Though the number is small (vary from 3 in the study of negative Vs neutral to 6 in the study of positive Vs neutral), we kept these observations as outliers.

Table-1: Recognition rate $(\%)$ using various classification algorithms for two emotional category negative and positive with reference to its neutral states

\begin{tabular}{|c|c|c|c|}
\hline \multicolumn{2}{|c|}{$\begin{array}{c}\text { Types of Classification } \\
\text { Algorithms }\end{array}$} & $\begin{array}{c}\text { Negative Vs } \\
\text { Neutral }\end{array}$ & $\begin{array}{c}\text { Positive Vs } \\
\text { Neutral }\end{array}$ \\
\hline \multirow{4}{*}{ Rules } & Simple Logistics & $71.95 \%$ & $80.24 \%$ \\
\cline { 2 - 4 } & SMO & $67.07 \%$ & $62.66 \%$ \\
\cline { 2 - 4 } & $\begin{array}{c}\text { Multilayer } \\
\text { Perceptron }\end{array}$ & $79.26 \%$ & $71.6 \%$ \\
\hline \multirow{4}{*}{ Trees } & Random Tree & $85.36 \%$ & $75.30 \%$ \\
\cline { 2 - 4 } & J48 & $84.14 \%$ & $88.88 \%$ \\
\cline { 2 - 4 } & BF Tree & $89.02 \%$ & $86.41 \%$ \\
\hline
\end{tabular}

\section{CONCLUSION}

It has been observed that approximately $70 \%$ of users are following the trend of decrease in the typing speed in negative emotional state while interacting with the keyboard with reference to its corresponding neutral state. One reason for this could be that negative states include such as restless, irritated, contempt etc. not all of these may have similar influence on typing speed. And approximately $83 \%$ of the users are showing the trend of increase in the typing speed in positive emotional state with reference to the neutral state. We couldn't find any generalization of the typing speed trend with the emotional categories.

\section{FUTURE WORK}

Interaction with keyboard varies from user to user and one of the causes may be their emotional states. And we see some sign to be able to differentiate between positive and negative emotional state of the user during his interaction with keyboard. One major limitation of such a system lies in the input given by the participants themselves while typing in the editor. They were expected to know their emotional states while typing. Another limitation is the sample size which has to be increased. Though we had done our experiments on small database (having 40 subjects, each has given test for three times), we get some cue relating to emotional state during user's interaction with keyboard. The current work provides some leads to investigate this issue further, by considering a large sample size and enhancing the reliability of estimating the user emotional states.

In the future, we extend our studies to the usage of Self Assessment Manikin (SAM) to collect the input from the users for measuring their emotions (Bradley and Lang, 1994). SAM is a pictorial scale allowing for direct ratings of arousal, valence and dominance. One more provision could be using movie clipping correspondingly to the emotional category. These modification and exploration of other properties as cues are currently in progress. 
Figure- 3: Variation of typing speed for negative and neutral emotional state across various users

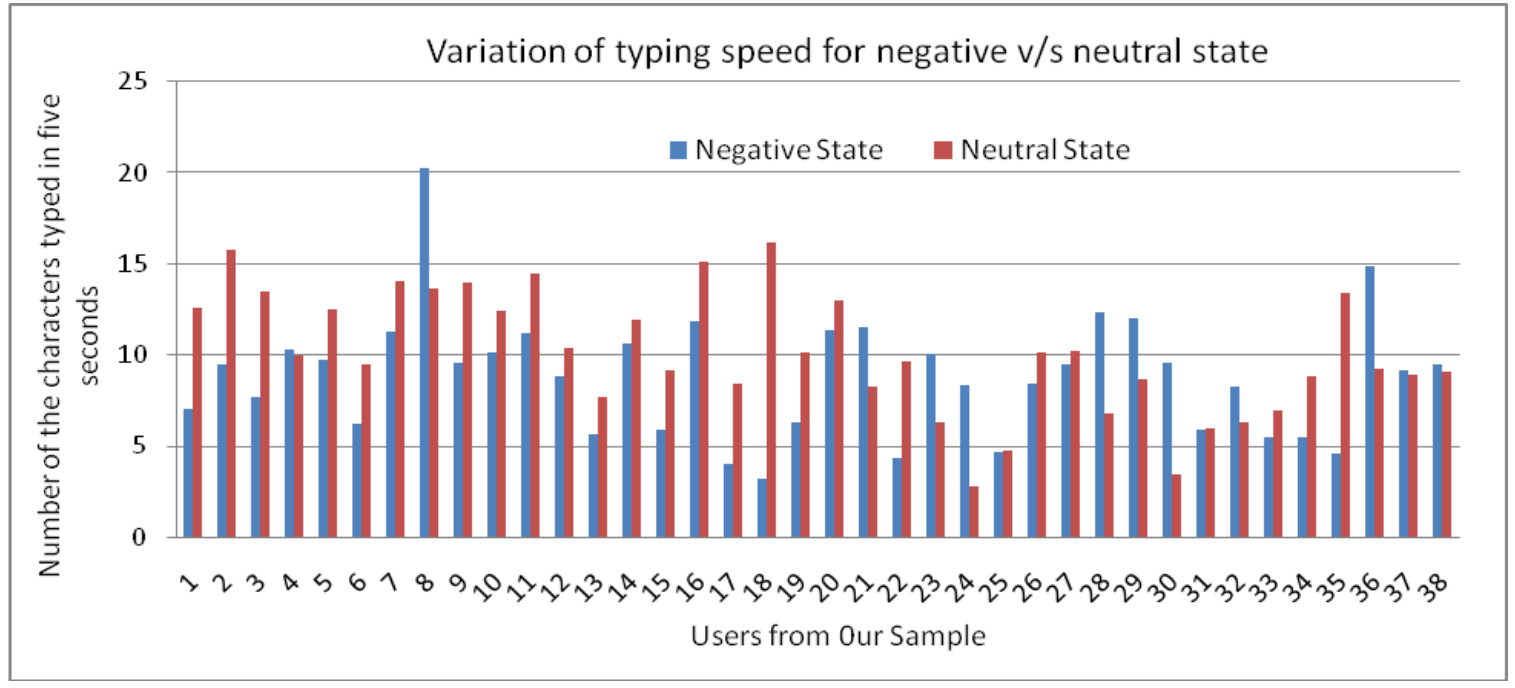

Figure- 4: Variation of typing speed for positive and neutral emotional state across various users

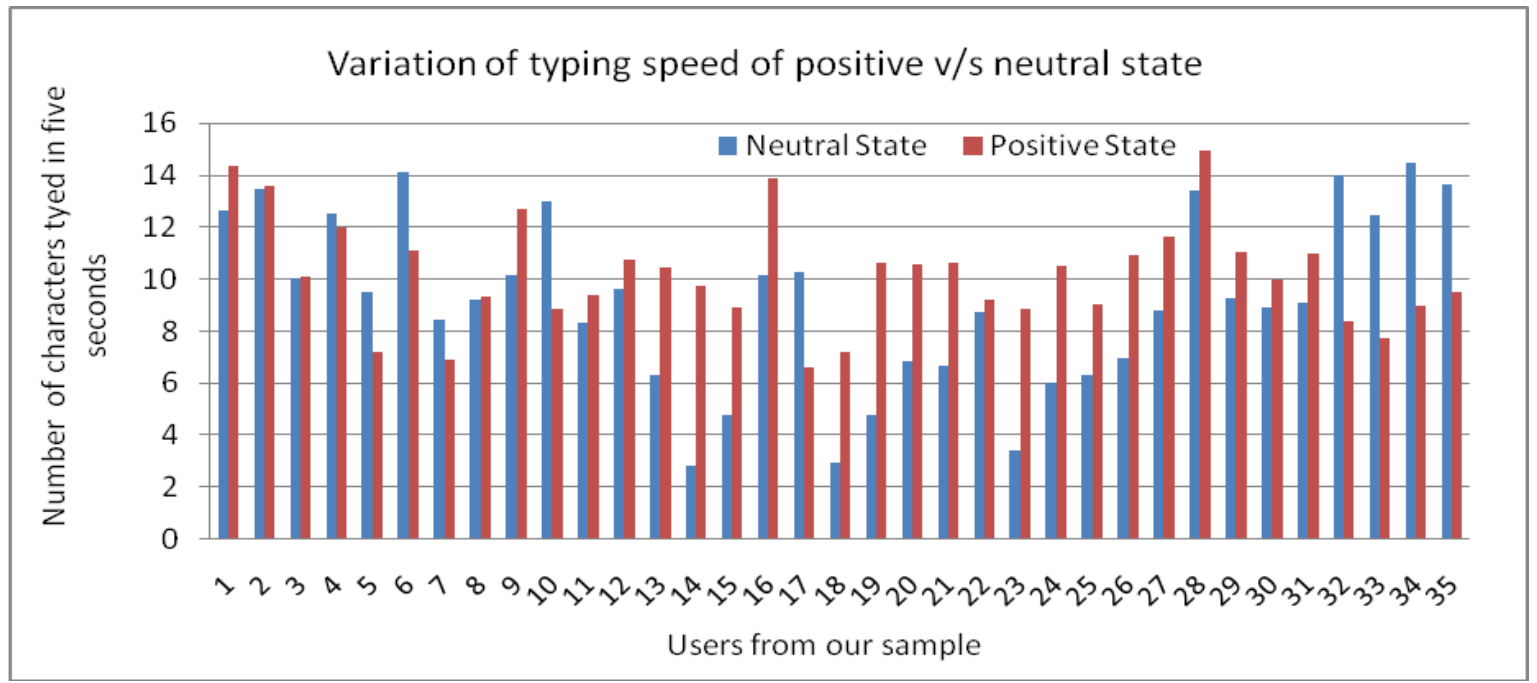

\section{REFERENCES}

[1] Ekman, P. 1982. Emotion in the human face. New York: Cambridfe University Press.

[2] Ekman, P. 1992. An argument for basic emotions. Cognition and Emotion, 6(3/4), p.169-200.

[3] Ekman, P. and Davidson, R.J. 1994. The Nature of Emotion Fundamental Questions: Oxford University Press Inc.

[4] Ekman, P. and Friesen, W.V. 1977. Facial action coding system. Consulting Psychologists Press.
[5] Ekman, P. and Friesen, W.V. 1975. Unmasking the face. A guide to recognizing emotions from facial clues. Englewood Cliffs, New Jersey: Prentice-Hall.

[6] Oviatt, S. 2003. User-modeling and evaluation of multimodal interfaces. Proceedings of the IEEE, Institute of Electrical and Electronics Engineers, p. 1457-1468.

[7] Picard, R.W. and Klein, J. 2002. Computers that recognize and respond to user emotion: theoretical and practical implications. Interacting with Computers 14(2), p. 141169.

[8] Wu, Y. and Huang, T. S. 2001. Hand modeling, analysis and recognition for vision based human computer 
interaction. IEEE Signal Processing Magazine 18(3) p: 5160.

[9] Zimmermann, P., Guttormsen, S., Danuser, B., Gomez, P.2003. Affective computing-a rationale for measuring mood with mouse and keyboard. International journal of occupational safety and ergonomics: JOSE vol. 9, issue 4, p. 539-551.

[10] De Silva, L., Miyasato, T., Nakatsu, R. 1997. Facial Emotion recognition using multimodal information, in Proc. IEEE Int. Conf. on Information, Communications and Signal Processing (ICICS'97) p. 397-401.

[11] Virvou, M., Tsihrintzis, G.A., Alepis, E., Stathopoulou, I.O., Kabbassi, K. 2007. Combining Empirical Studies of Audio-Lingual and Visual-Facial Modalities for Emotion Recognition. Lecture Notes in Computer Science, Volume 4693, p. $1130-1137$

[12] Picard, R.W. 2003. Affective Computing: Challenges, Int. Journal of Human-Computer Studies, Vol. 59, Issues 1-2, p. 55-64.
[13] Busso, C., Deng, Z., Yildirim, S., Bulut, M., Lee, C.M.,Kazemzadeh, A., Lee, S., Neumann, U., Narayanan, S. 2004. Analysis of emotion recognition using facial expressions, speech and multimodal information, Proceedings of the 6th international conference on Multimodal interfaces, State College, PA, USA. p. 205 211.

[14] Pantic, M., Rothkrantz, L.J.M. 2003. Toward and Affectsensitive multimodal human-computer interaction. Vol. 91, Proceedings of the IEEE. p. 1370-1390.

[15] George A. Tsihrintzis, Maria Virvou, Efthymios Alepis, Ioanna-Ourania Stathopoulou, 2008. Towards Improving Visual-Facial Emotion Recognition through Use of Complementary Keyboard-Stroke Pattern Information. itng, pp.32-37, Fifth International Conference on Information Technology: New Generation. 